\title{
Effect of varying material anisotropy on critical current anisotropy in vicinal $\mathrm{YBa}_{2} \mathrm{Cu}_{3} \mathrm{O}_{7-\delta}$ thin films
}

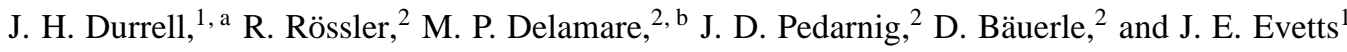 \\ ${ }^{I}$ Department of Materials Science and Metallurgy and IRC in Superconductivity, \\ University of Cambridge, Pembroke Street, Cambridge, CB2 3QZ, UK. \\ ${ }^{2}$ Angewandte Physik, Johannes-Kepler-Universität Linz, A-4040 Linz, Austria
}

\begin{abstract}
The high $T_{c}$ cuprate superconductors are noted for their anisotropic layered structure, certain of these materials indeed tend toward the limit of a Lawrence-Doniach superconductor. However, $\mathrm{YBa}_{2} \mathrm{Cu}_{3} \mathrm{O}_{7-\delta}$ has a smaller anisotropy than would be expected from its interlayer spacing. This is due to the cuprate chains in the structure. To investigate the influence of the chain oxygen on transport properties critical current versus applied field angle measurements were performed on fully oxygenated and de-oxygenated $\mathrm{YBa}_{2} \mathrm{Cu}_{3} \mathrm{O}_{7-\delta}$ thin films and optimally oxygenated $\mathrm{Y}_{0.75} \mathrm{Ca}_{0.2} \mathrm{Ba}_{2} \mathrm{Cu}_{3} \mathrm{O}_{7-\delta}$ thin films. The films were grown on $10^{\circ}$ mis-cut $\mathrm{SrTiO}_{3}$ substrates to enable the intrinsic vortex channelling effect to be observed. The form of the vortex channelling minimum observed in field angle dependent critical current studies on the films was seen to depend on film oxygenation. The vortex channelling effect is dependent on a angular dependent cross-over to a string-pancake flux line lattice. The results obtained appear to be consistent with the prediction of Blatter et al. [Rev. Mod. Phys., 66 (4): 1125 (1994)] that increased superconducting anisotropy leads to the kinked string-pancake lattice existing over a smaller angular range.
\end{abstract}

Among the cuprate high $T_{c}$ superconductors $\mathrm{YBa}_{2} \mathrm{Cu}_{3} \mathrm{O}_{7-\delta}$ (YBCO) is of particular interest due to its relatively low anisotropy parameter. For example while, $d$, the cuprate plane spacing is $1.2 \mathrm{~nm}$ in YBCO as compared to $1.5 \mathrm{~nm}$ in $\mathrm{BSCCO}$ [1], values for the superconducting mass anisotropy parameter $\gamma^{2}=\left(m_{c} / m_{a}\right)$ are quoted as 5-7 for YBCO and 50-200 for $\mathrm{Bi}_{2} \mathrm{Sr}_{2} \mathrm{Ca}_{1} \mathrm{Cu}_{2} \mathrm{O}_{8+x}$ [2, 3, 4, 5] (the large uncertainty in the $\mathrm{Bi}_{2} \mathrm{Sr}_{2} \mathrm{Ca}_{1} \mathrm{Cu}_{2} \mathrm{O}_{8+x}$ value is due to the difficulty in measuring its $c$ axis transport properties).

The structure of YBCO contains 'cuprate chains' inside the blocking layers between the cuprate planes associated with superconductivity. In most cuprate superconductors the blocking layers act as an insulating charge reservoir, in YBCO however, the cuprate chains also have superconducting properties [6]. This is the feature of YBCO which leads to the lower anisotropy seen in YBCO as compared to that predicted on the basis of the interlayer spacing. The chain oxygen in YBCO is highly mobile, under doping or over doping of the material by moving the oxygenation away from $\delta \sim 0.08$ is associated with a reduction in $T_{c}$. A reduced oxygenation level will, however, also increase the superconducting anisotropy of the material by disrupting the cuprate chains. Importantly the removal of oxygen from the chains does not increase pinning [7, 8]. However, there is a definite increase in superconducting anisotropy with $\delta$ at larger values [9, 10], indeed Deak et al. [11] indicate that the anisotropy more than doubles for $\delta>0.2$.

Intrinsic vortex channelling along the $a-b$ planes has previously been reported in optimally doped YBCO films grown on mis-cut (vicinal) substrates [12]. The development of the channelling minima in these measurements has been attributed to the appearance of a kinked (string/pancake) vortex state as the angle, $\theta$, between the $a-b$ planes and the applied field is reduced. The pinning of vortex string elements against a Lorentz force directed parallel to the $a-b$ planes is relatively weak. By using a vicinal geometry thin film it is

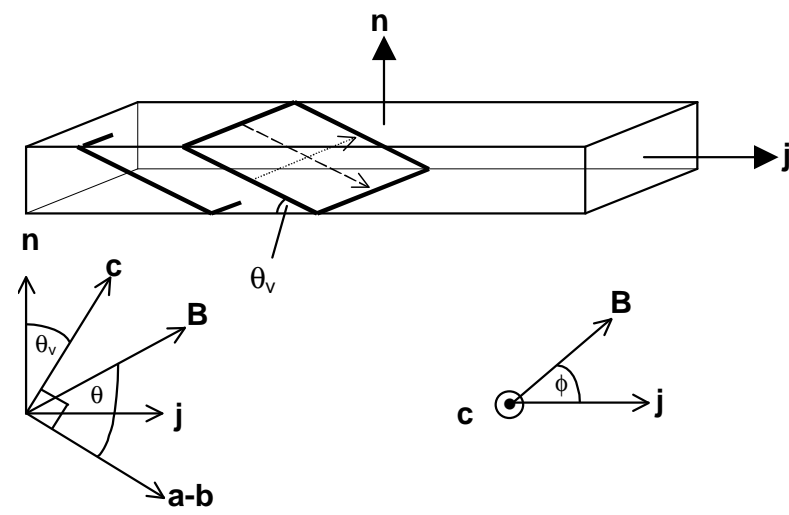

FIG. 1: Measurement geometry. The angle $\theta$ is that between the applied magnetic field and the $a-b$ crystallographic planes. The angle $\phi$ is that of the rotation of the applied field with respect to the direction of vicinal tilt, the magnitude of which is defined as $\theta_{v}$. The measurements reported here were carried out with $\phi=0$ so the directions of the normal to the film, $\mathbf{n}$, the $c$-axis, the field and the current all lay in the same plane.

possible to arrange for a component of the Lorentz force to act along the $a-b$ planes. This leads to a marked reduction in the critical current when the magnetic field is applied parallel to the $a-b$ planes. The kinked vortex state is intimately related to the anisotropy of the superconducting material. The variation of the structure of flux lines in YBCO as the angle, $\theta$, between the applied field and the $a-b$ planes varies has been discussed by Blatter et al. [5]. For temperatures below $T_{c r}$, where $T_{c r}$ is defined by $\epsilon \xi_{a b}\left(T_{c r}\right)=d / \sqrt{ } 2(\sim 80 \mathrm{~K}$ in optimally doped YBCO), the critical angle between the applied magnetic field and the $a-b$ planes required for the development of a fully kinked string-pancake structure is given by:

$$
\tan \left(\theta_{2}\right)=\epsilon
$$

where $\epsilon=1 / \gamma$ is the Ginzburg-Landau (G-L) anisotropy 

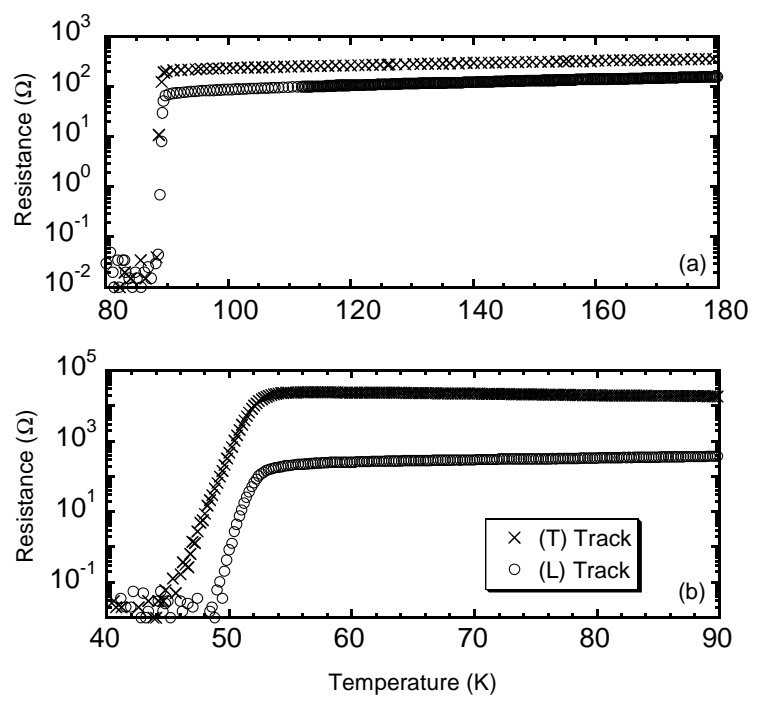

FIG. 2: Resistance versus temperature plots for the fully (a) and de-oxygenated (b) YBCO films. It can be seen that the in-plane resistivity anisotropy is greatly increased in the de-oxygenated film.

parameter. Between $\theta_{2}$ and a larger angle $\theta_{1}$ the flux lines exhibit a distorted structure while for $\theta>\theta_{1}$ they adopt the conventional rectilinear Abrikosov form. $\theta_{1}$ is defined as $\tan \left(\theta_{1}\right)=d / \epsilon_{a b}\left(T / T_{c}\right)$. From this it can be deduced that an increasing G-L anisotropy parameter is predicted to lead to a smaller angular range of kinked structure.

In order to study the effect of material anisotropy on the vortex channelling effect vicinal YBCO films with different anisotropies were prepared. The $\mathrm{YBa}_{2} \mathrm{Cu}_{3} \mathrm{O}_{7-\delta}$ and $\mathrm{Y}_{0.75} \mathrm{Ca}_{0.2} \mathrm{Ba}_{2} \mathrm{Cu}_{3} \mathrm{O}_{7-\delta}$ thin films were prepared under identical conditions on $10^{\circ}$ miscut vicinal (001) $\mathrm{SrTiO}_{3}$ substrates by pulsed-laser deposition [13]. The film were $\sim 200 \mathrm{~nm}$ thick. In situ post-annealing of the films wa $\mathrm{s}$ performed at an oxygen pressure of 800 mbar and 0.7 mbar for the fully oxygenated and de-oxygenated YBCO films respectively. The Ca-substited film was annealed at $\sim 15$ mbar for optimum critical temperature [14]. Tracks were patterned onto the films using photolithography and Ar ion milling to give tracks $100 \mu \mathrm{m}$ by $10 \mu \mathrm{m}$ both parallel, $\mathbf{L}$, and perpendicular, $\mathbf{T}$, to the vicinal steps. Critical current values were determined from IV characteristics using a $0.55 \mu \mathrm{V}$ criterion, measurements were performed using a two-axis goniometer mounted in a $8 \mathrm{~T}$ magnet. The experimental geometry is shown in Figure 11 The resistive transition for both films was measured on both the $\mathbf{T}$ and $\mathbf{L}$ tracks. As discussed by Zahner et al. [15] it is possible to deduce the resistance anisotropy between the $a-b$ and the $c$ axes from the resistivity ratio between the $\mathbf{T}$ and $\mathbf{L}$ tracks, $\gamma_{T L}$ : $\gamma_{\rho}=\left[\gamma_{T L}-\cos ^{2}\left(\theta_{v}\right)\right] / \sin ^{2}\left(\theta_{v}\right)$, where $\theta_{v}$ is the vicinal mis-cut angle. The resistance temperature data shown in Fig. 2 indicates a resistivity anisotropy at $100 \mathrm{~K}$ of 66 for the optimally oxygenated film and 1600 for the de-oxygenated film. These values are consistent with those observed in single crystal samples [1].

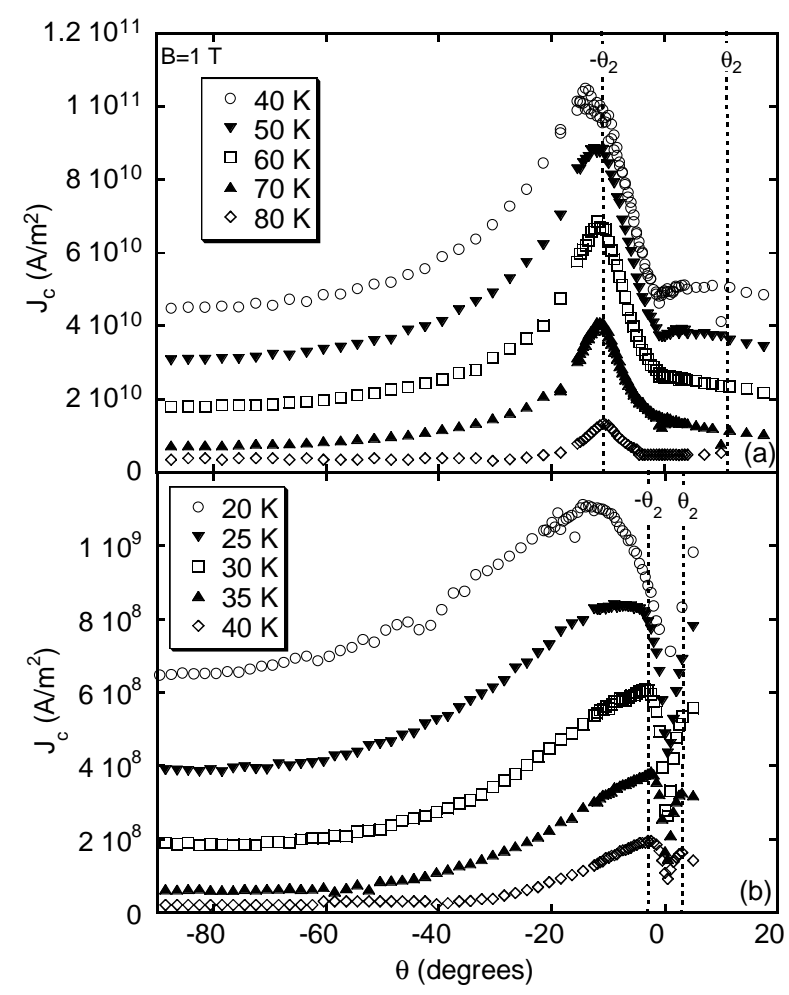

FIG. 3: $J_{c}(\theta)$ plots for the fully (a) and de-oxygenated (b) films measured. These data were obtained in the $\phi=0$ geometry so the channelling minima and the 'force-free' peak are offset by the vicinal angle, $10^{\circ}$. The dotted lines indicate the predicted values of $\theta_{2}$.

The $T_{c}$ values were $90 \mathrm{~K}$ and $51 \mathrm{~K}$ for the fully- and deoxygenated films respectively. Although other factors apart from $\delta$ affect $T_{c}$ this value of $T_{c}$ suggests a $\delta$ value of about 0.4 [16] for the de-oxygenated film. From Eq. 1 and the relationship between $\delta$ and $\gamma$ found by Deak et al. [11] this would predict, at $t=0$, a value for $\theta_{2}$ of $3^{\circ}$ as compared to $11^{\circ}$ for fully oxygenated YBCO. Furthermore $T_{c r}$ becomes closer to $T_{c}$ with increasing anisotropy, this means the channelling minimum should be visible in $J_{c}(\theta)$ measurements over a wider range of reduced temperature in the de-oxygenated sample.

Figure 3 shows the form of the vortex channelling effect at a magnetic field of 1 Tesla and various temperatures for the fully oxygenated and de-oxygenated films measured. The results obtained for the fully oxygenated sample are, as would be expected, very similar to those previously reported [12]. From the data presented in Fig. 3 it is clear that there is a distinct change in the form of the channelling minima for the deoxygenated film. As would be expected the angular range over which a departure from the conventional truncated $1 / \cos \theta$ force free behavior appears to be smaller for the case of the de-oxygenated film. As the films were grown under identical conditions, apart from the oxygen partial pressure during annealing, this change in the $J_{c}(\theta)$ behavior is reasonably associated with a change in the anisotropy of the sample studied. 


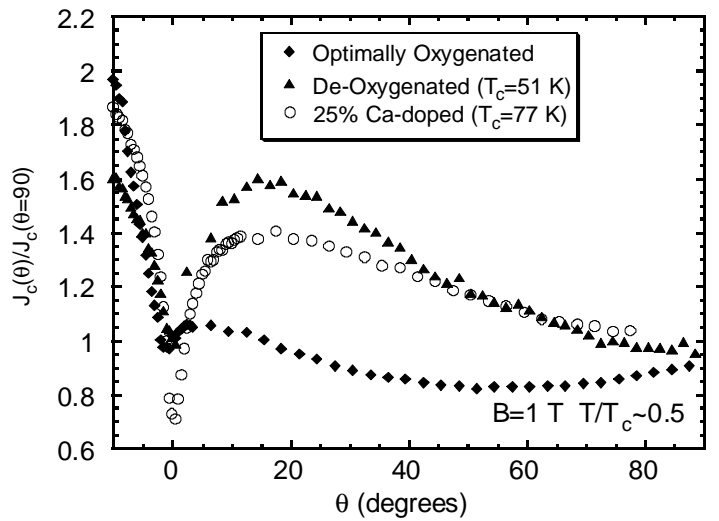

FIG. 4: $J_{c}(\theta) / J_{c}(\theta=90)$ data plotted for all three films. These data was taken at $1 \mathrm{~T}$ and the same reduced temperature.

Calcium doping has been extensively studied as a means of improving the properties of grain boundaries in YBCO. In the grain, however, in order to obtain a high $T_{c}$ a reduced chain oxygen occupancy is required as the $\mathrm{Ca}$ doping shifts the peak in the $T_{c}$ versus $\delta$ curve. $25 \%$ Ca-substituted YBCO with an oxygenation corresponding to optimum $T_{c}$ will therefore have oxygen vacancies in its cuprate chains and would be expected therefore to exhibit a stronger superconducting anisotropy.

A comparison of the $J_{c}(\theta)$ behavior of a Ca-doped film with a fully- and de-oxygenated film is shown in Figure 4 The Ca-doped film was grown in the same PLD system and under similar conditions, but with a $25 \%$ Ca-substituted target. It is interesting to note that the Ca-doped film exhibits broadly similar behavior to the de-oxygenated film. This is evidence that $\mathrm{Ca}$-doped YBCO also exhibits an increased superconducting anisotropy and that this is due to the oxygen vacancies in the cuprate chain needed to obtain an optimum $T_{c}$. From the measurements presented in this letter it is clear that the oxygenation of the cuprate planes strongly affects the superconducting anisotropy of the material. A variation of this anisotropy changes the way the structure of individual flux lines evolves as the applied magnetic field is rotated with respect to the cuprate planes. This anisotropy dependent change is reflected in the vicinal channelling effect observed in YBCO samples with differing chain oxygen concentration. This increased anisotropy is also apparent in Ca-doped YBCO which, for optimum $T_{c}$, must have a lower oxygenation than non Ca-doped YBCO.

a Electronic address: jhd25@cam.ac.uk

b Now at: CNRT Matériaux de Basse-Normandie / ENSICAEN, 6 Boulevard Maréchal Juin, 14050 CAEN cedex 4, France

[1] T. Datta, in Concise Encylopedia of Magnetic and Superconducting Materials, edited by J. E. Evetts (Pergamon, Oxford, 1992).

[2] D. E. Farrell, S. Bonham, J. Foster, Y. C. Chang, P. Z. Jiang, K. G. Vandervoort, D. J. Lam, and V. G. Kogan, Physical Review Letters 63, 782 (1989).

[3] N. Chikumoto, M. Konczykowski, N. Motohira, and K. Kishio, Physica C 199, 32 (1992).

[4] J. C. Martinez, S. H. Brongersma, A. Koshelev, B. Ivlev, P. H. Kes, R. P. Griessen, D. G. de Groot, Z. Tarnavski, and A. A. Menovsky, Physical Review Letters 69, 2276 (1992).

[5] G. Blatter, M. V. Feigelman, V. B. Geshkenbein, A. I. Larkin, and V. M. Vinokur, Reviews of Modern Physics 66, 1125 (1994).

[6] J. L. Tallon, C. Bernhard, U. Binninger, A. Hofer, G. V. M. Williams, E. J. Ansaldo, J. I. Budnick, and C. Niedermayer, Physical Review Letters 74, 1008 (1995).

[7] D. K. Christen and R. Feenstra, Physica C 185, 2225 (1991).

[8] J. G. Ossandon, J. R. Thompson, D. K. Christen, B. C. Sales, H. R. Kerchner, J. O. Thomson, Y. R. Sun, K. W. Lay, and J. E. Tkaczyk, Physical Review B 45, 12534 (1992).

[9] R. J. Cava, A. W. Hewat, E. A. Hewat, B. Batlogg, M. Marezio, K. M. Rabe, J. J. Krajewski, W. F. Peck, and L. W. Rupp, Physica C 165, 419 (1990).

[10] L. F. Hou, J. Deak, P. Metcalf, and M. McElfresh, Physical Review B 50, 7226 (1994).

[11] J. Deak, L. F. Hou, P. Metcalf, and M. McElfresh, Physical Review B 51, 705 (1995).

[12] P. Berghuis, E. DiBartolomeo, G. A. Wagner, and J. E. Evetts, Physical Review Letters 79, 2332 (1997).

[13] D. Bäuerle, Laser Processing and Chemistry (Springer, New York, 2000), 3rd ed.

[14] M. P. Delamare, R. K. Schöppl, J. Pedarnig, and D. Bäuerle, Physica C 372-376, 638 (2002).

[15] T. Zahner, R. Stierstorfer, R. Rössler, J. D. Pedarnig, D. Bäuerle, and H. Lengfellner, Physica C 298, 91 (1998).

[16] M. Cyrot and D. Pavuna, Introduction to Superconductivity and High-Tc materials (World Scientific, Singapore, 1995). 